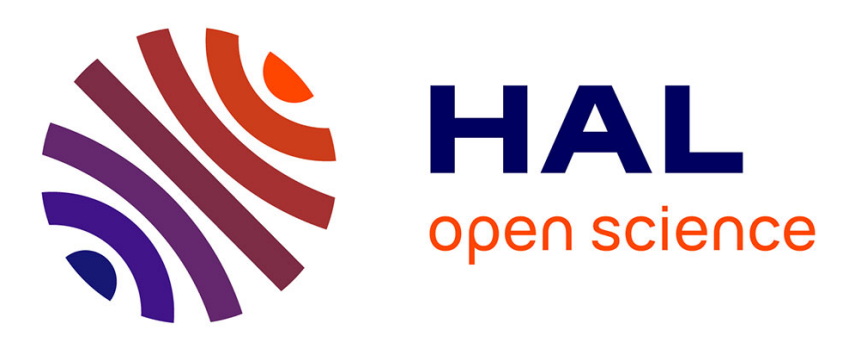

\title{
Infection of the Biomphalaria glabrata vector snail by Schistosoma mansoni parasites drives snail microbiota dysbiosis
}

Anaïs Portet, Eve Toulza, Ana Lokmer, Camille Huot, David Duval, Richard Galinier, Benjamin Gourbal

\section{To cite this version:}

Anaïs Portet, Eve Toulza, Ana Lokmer, Camille Huot, David Duval, et al.. Infection of the Biomphalaria glabrata vector snail by Schistosoma mansoni parasites drives snail microbiota dysbiosis. 2018. hal-03134818

\author{
HAL Id: hal-03134818 \\ https://hal.science/hal-03134818
}

Preprint submitted on 11 Feb 2021

HAL is a multi-disciplinary open access archive for the deposit and dissemination of scientific research documents, whether they are published or not. The documents may come from teaching and research institutions in France or abroad, or from public or private research centers.
L'archive ouverte pluridisciplinaire HAL, est destinée au dépôt et à la diffusion de documents scientifiques de niveau recherche, publiés ou non, émanant des établissements d'enseignement et de recherche français ou étrangers, des laboratoires publics ou privés. 


\section{Infection of the Biomphalaria glabrata vector snail by Schistosoma mansoni}

\section{2 parasites drives snail microbiota dysbiosis.}

3

4 Anaïs Portet ${ }^{1}$, Eve Toulza ${ }^{1}$, Ana Lokmer ${ }^{2}$, Camille Huot $^{1}$, David Duval ${ }^{1}$, Richard Galinier ${ }^{1}$ and 5 Benjamin Gourbal ${ }^{1, \S}$

6

$7 \quad{ }^{1}$ IHPE, Univ. Montpellier, CNRS, Ifremer, Univ. Perpignan Via Domitia, Perpignan France

$9{ }^{2}$ Department Coastal Ecology, Wadden Sea Station Sylt, Alfred Wegener Institute, Helmholtz 10 Centre for Polar and Marine Research, List/Sylt, Germany.

11

12 AP: ap2133@cam.ac.uk, ET: eve.toulza@univ-perp.fr, AL: Ana.Lokmer@awi.de, CH:

13 camillehuot86@gmail.com, DD: david.duval@univ-perp.fr, RG: richard.galinier@univ-perp.fr,

14 BG: benjamin.gourbal@univ-perp.fr

$16 \S$ Corresponding author :

17 Benjamin GOURBAL

18 UMR5244 IHPE, Université de Perpignan

1958 avenue Paul Alduy, 66860 Perpignan

20 benjamin.gourbal@univ-perp.fr

21

22 Running title: Biomphalaria glabrata snail microbiota dysbiosis 


\section{Summary}

25 Host-associated microbiota can affect the fitness of its host in a number of ways, including the

26 modification of host-parasite interactions and thus the outcome of disease. Biomphalaria

27 glabrata is the vector snail of the trematode Schistosoma mansoni, the agent of human

28 schistosomiasis, causing hundreds of thousands of deaths every year. Here, we present the first

29 study of the snail bacterial microbiota in response to Schistosoma infection. To examine the

30 interplay between B. glabrata, S. mansoni and snail microbiota, snails were infected and the

31 microbiota composition was analysed by massive 16S rDNA amplicon sequencing approach. We

32 characterized the Biomphalaria bacterial microbiota at the individual level in both naïve and

33 infected snails. Sympatric and allopatric strains of parasites were used for infections and re-

34 infections to analyse the modification or dysbiosis of snail microbiota in different host-parasite

35 co-evolutionary contexts. Concomitantly, using RNAseq data, we investigated the link between

36 bacterial microbiota dysbiosis and snail anti-microbial peptide immune response. This work

37 paves the way for a better understanding of snail/schistosome interaction, and would have

38 critical consequences in terms of snail control strategies for fighting schistosomiasis disease in 39 the field.

42 Key words: microbiota; bacteria; Biomphalaria snail; Schistosoma infection; immune response;

43 dysbiosis 


\section{Introduction}

Several studies have shown that the microbiota interacts with pathogens and/or host immunity in invertebrates. For example, the midgut microbiota of the mosquito Aedes sp. elicits a basal immune activity of the immune system (Ramirez et al., 2012) mostly by activating Toll pathways (Xi et al., 2008). In Anopheles gambiae, some members of its microbiota can limit malaria transmission, by inducing a wide antimicrobial immune response (Gendrin, 2017). More precisely, bacteria of the genus Enterobacter, found in the mosquito microbiota, were shown to produce reactive oxygen species (ROS) that inhibit Plasmodium development (Cirimotich et al., 2011). Furthermore, the endosymbiotic bacteria Wolbachia induce expression of immune genes, like TEP1 (Thioester-containing protein 1), LRIM1 (Leucine-Rich Immune Molecule 1) or defensin 1 (Joshi et al., 2017). In Drosophila, the bacterial microbiota is necessary to produce the Pvf2, a PDGF/VEGF-like growth factor that restricts enteric viral infection (Sansone et al., 2015). The microbiota may also play a role in immune priming. For example, in Anopheles gambiae, hemocyte priming during the Plasmodium infection is naturally induced by the gut microbiota, whose absence results in more severe infections and re-infections (Rodrigues et al., 2010). Similarly, the gut microbiota is necessary for immune priming in Tribolium castaneum and efficient response against Bacillus thuringiensis for Tenebrionis (Futo et al., 2015). Finally, host immunity can influence the tolerance or control the microbiota. In the tick Ixodes scapularis, the protein PIXR, secreted by the tick's gut, inhibits the bacterial biofilm formation and supports gut eubiosis (healthy gut microbiota), its inactivation leading to dysbiosis. This change in gut microbiota facilitates the colonization by Borrelia burgdorferi, the Lyme disease agent (Narasimhan et al., 2017). In Drosophila, for example, the intestinal homeobox gene Caudal regulates the commensal-gut mutualism by repressing nuclear factor kappa B- 
68 dependent antimicrobial peptide genes (Ryu et al., 2008). In Hydra, the innate immune sensors

69 and effectors protect not only against pathogens but also control microbiota homeostasis

70 (Augustin et al., 2010). Moreover, host specific antimicrobial peptides (Fraune et al., 2010;

71 Franzenburg et al., 2012) and a rich repertoire of pattern recognition receptors (Bosch, 2013)

72 are involved in maintaining homeostasis between the host and the resident microbiota.

73 Similarly, bactericidal permeability-increasing proteins (BPIs) shape bacterial communities in

74 the light organ of the squid Euprymna scolopes and prevent their spill over to other tissues

75 (Chen et al., 2017).

76 Biomphalaria glabrata is a freshwater snail (Lophotrochozoa, Planorbidae), living in inter-

77 tropical regions of Latin America, in rivers, ponds, waterways and other freshwater

78 environments. B. glabrata snails have important medical and epidemiological impacts due to

79 their role as the main vector of Schistosoma mansoni (Lophotrochozoa, Platyhelminthes,

80 Trematoda), the agent of intestinal schistosomiasis. Schistosomiasis is the second most

81 widespread human parasitic disease after malaria, affecting over 200 million people worldwide

82 and causing 200000 deaths annually (WHO, 2002). The Schistosoma adult parasites mate in the

83 human host venous system. Female worms produce eggs that cross endothelial mesenteric

84 vessels and intestinal epithelium to reach faeces and finally the aquatic environment. Once in

85 the water, eggs hatch and release miracidia, the free-living snail-infective parasite stage. At the

86 following step of the life cycle, the miracidium needs to infect the freshwater snail B. glabrata.

87 Intensive asexual multiplication in the snail tissues leads to the continuous production of

88 hundreds of generations of cercariae, the free-living human-infective parasite stage.

89 At present, there is no effective vaccine against schistosomiasis and the treatment relies on a

90 single chemotherapeutic treatment, the Praziquantel (Doenhoff et al., 2009), against which 
91 resistance has been observed (Fallon and Doenhoff, 1994). Molluscicides have been also used

92 to impair Schistosoma transmission in the field. However, the dramatic effects of molluscicides

93 on natural environments prompt us to seek new ways to prevent and / or control this disease in

94 the field (Tennessen et al., 2015). We propose that a better understanding of ecological

95 interactions between, Schistosoma mansoni, Biomphalaria glabrata snails and its associated

96 microbiota might represent an interesting approach in search for alternative control strategies.

97 In this context, we have conducted numerous studies on immunological interactions

98 between the snail and the parasite (Mitta et al., 2005; Bouchut et al., 2008; Moné et al., 2011;

99 Coustau et al., 2015; Galinier et al., 2017; Mitta et al., 2017). We have demonstrated that the

100 nature of the snail immune response depends on the strain/species of host or of parasite

101 considered. Depending on both the host and the parasite intrinsic capacities, parasites either

102 develop normally in snail tissue (compatible interaction) or are encapsulated by hemocytes (the

103 snail immune cells) (incompatible interaction) (Mone et al., 2010; Mitta et al., 2012; Coustau et

104 al., 2015) (Fig. 1). Depending on the ecological context, totally different immunobiological

105 interactions have been described in B. glabrata/S. mansoni interactions. If immunosuppression

106 occurs during a sympatric interaction, an activation of the immune response is observed during

107 an allopatric interaction (Portet et al., 2019). Moreover, following primo-infection and

108 successive challenges, a shift from a cellular immune response toward a humoral immune

109 response has been described (Pinaud et al., 2016) (Fig. 1).

110 However, in the present host-parasite model, only few studies examined the bacterial

111 microbiota of Biomphalaria glabrata so far (Ducklow et al., 1979; Silva et al., 2013). The study

112 of aerobic heterotrophic cultivable flora of 200 snails (Ducklow et al., 1979) revealed five 113 predominant bacterial genera including Pseudomonas, Acinetobacter, Aeromonas, Vibrio and 
114 Enterobacter. In a more recent study, the Biomphalaria glabrata bacterial microbiota has been

115 characterized using 16 S rRNA sequences (Silva et al., 2013) of cultured isolates, identifying six

116 additional bacteria genera: Citrobacter, Cupriavidus, Rhizobium, Stenotrophomonas, Klebsiella

117 and Sphingomonas. In addition, diversity and composition of bacterial microbiota differed

118 between resistant and susceptible Biomphalaria phenotypes, with differences in relative

119 abundances of Gemmatimonas aurantiaca and Micavibrio aeruginosavorus. This observation

120 supports the potential link between the resistance against Schistosoma parasite and the

121 structure of snail-associated microbiota (Allan et al., 2018). However, no studies have examined

122 changes in bacterial microbiota of Biomphalaria snails by culture-independent methods nor

123 following Schistosoma infections so far.

124 Taking into account all the peculiarities of this system, the aim of the present study was to

125 investigate variation of microbiota in snail populations as well as to assess the influence of

126 varying immune response against Schistosoma on the host microbiota composition and

127 dynamics. To reach this goal, we first characterized the bacterial microbiota of naive

128 Biomphalaria glabrata snails using 16S rDNA metabarcoding. Then, we analysed changes in

129 microbiota composition following, (i) primo-infections with sympatric and allopatric S. mansoni

130 parasite isolates displaying the same prevalence and intensity phenotypes and, (ii) subsequent

131 secondary challenges with homologous or heterologous parasite strains. As snail immune

132 response strongly differs between such diverse snail/parasite combinations, we concomitantly

133 analysed the snail immune response using a massive sequencing transcriptomic approach

134 (RNAseq) in order to link it with the observed changes in the composition and diversity of the 135 microbiota. 


\section{Results}

\section{Characterization of healthy B. glabrata microbiota}

139 To examine the stability of naive BgBRE snail microbiota, we first inspected the microbiota

140 diversity and composition in the control naive snails at day 0 and 25 of the experiment. We

141 found no significant differences between the BO and B25 snails in any of the alpha diversity 142 indices (Table S3, Table S4).

$143 \quad$ Naive snails showed little inter-individual variability and a stable composition at the 144 phylum level over time (Fig. 2A, Table S3, Table S4), with Proteobacteria, Bacteroidetes, 145 Cyanobacteria and Planctomycetes phyla being the most represented (Fig 2A). Moreover, the 146 bacterial microbiota of naïve BgBRE snails displayed a considerable temporal and inter147 individual stability at the family level (Fig. 2B). The stability of naive snail microbiota 148 composition over time (Fig. 2) could be related to the laboratory rearing conditions and 149 laboratory environmental abiotic variables (water composition, temperature, $\mathrm{pH}$, food) that 150 were tidily controlled and that did thus not result in changes in host snail physiology or 151 metabolism.

152 In terms of composition, we observed that 67 (69\%) and 86 (89\%) out of 97 identified

153 families were shared by all individuals of B0 or B25 naive snails respectively (Fig. 2B). Those 154 results were used to determine the core microbiota. We defined core microbiota as the families

155 that were present in $100 \%$ of the naive snails. Applying this definition, we identified 62 out of 15697 families found in all naive individual snails ( $\mathrm{BO}$ and B25 altogether), and thus constituting the 157 B. glabrata core-microbiota (Table S7 and S8). 
160 After establishing the stability of naive Biomphalaria bacterial microbiota, we investigated

161 whether Schistosoma mansoni infections affected the microbiota structure and dynamics.

162 To investigate the influence of parasite infections on the bacterial microbiota, we analysed

163 microbiota dynamics following sympatric or allopatric primo-infections and homologous or

164 heterologous challenges (Fig. 1).

166 We did not observe any significant changes in alpha diversity during the course of primo-

167 infection compared to naive snails, excepted a decrease in the Shannon's H diversity index at

168 the fourth day after sympatric infection (Fig. 3, Table S3, Mann-Whitney U test: Table S4).

169 Conversely, all indices changed significantly following homologous or heterologous challenges

170 (Fig. 3, Table S3, Table S4). Indeed, the observed species richness (Mann-Whitney $U$ test,

$171 \mathrm{p}=0.002$ ), the Chao 1 richness index (Mann-Whitney $U$ test, $p=0.006$ ), Shannon diversity index

$172(p=0.0004)$ and Pielou evenness index (Mann-Whitney $U$ test, $p=0.009$ ) were significantly

173 reduced following homologous or heterologous challenges compared to naive and primo-

174 infected snails (Fig. 3, Table S3, Table S4). However, the observed drop in alpha diversity

175 disappeared by the fourth day after heterologous challenge (Fig. 3, Table S4). In addition, alpha

176 diversity was mainly affected by the challenge, regardless of its type (homologous or

177 heterologous). The primo-infection did not significantly affect alpha diversity except for the

178 sympatric combination at day 4 after infection (Table S4).

179 Regarding the beta diversity, Principal Coordinate Analysis (PCoA) of Bray-Curtis dissimilarities

180 revealed that $\mathrm{BB} 25$ samples grouped together with the naive snail samples (BO and $\mathrm{B} 25$ ) and

181 were separated from the infected-snail samples along both axes (Fig. 4A, Table S5). However,

182 the BB25 samples were significantly different from the naive snail samples (Table S5). The fact 
183 that, BB25 grouped with naive snails rather than with infected-snail samples suggests that the

184 snail microbiota is resilient to infection, with a tendency to recover between day 4 and day 25

185 after the primo-infection (Fig. 4A). Analysis of Bray-Curtis dissimilarity index revealed a

186 significant difference between naive and primo-infected samples $(p=0.001)$ and also between

187 naive and challenged samples ( $p=0.001$ ) (Table S5). Concerning the infected-snail samples, all

188 experimental samples were significantly different from each other with the exception of BB1

189 versus BB4 and BBB1 versus BBV1 (Table S5).

190 In addition, the second PcoA axis separated the samples according to the course of infection

191 (i.e. the day 1 from the day 4 samples) (Fig. 4A), whereas the day 1 after-challenge (BBB1,

192 BBV1), the day 1 after-primo-infection (BB1, BV1) and all of the day 4 infection samples (BB4,

193 BV4, BBB4, BBV4) were separated along the first axis (Fig. 4A). Interestingly, day 1 after-

194 challenge samples (BBB1, BBV1) were more different from the naive microbial communities

195 than the day 1 primo-infection samples (BB1, BV1). Moreover, the second axis separated the

196 sympatric primo-infection (BB1) from the allopatric primo-infection (BV1) (Fig. 4A). Finally, all

197 day 4 samples grouped together reflecting the similarity between these samples regardless of

198 experimental infection conditions (Fig. 4A). Even if the day of infection appears as the main

199 explaining factor, these results indicate that the snail microbiota response to the infection also

200 depends on the type of infection (sympatric vs. allopatric or primo-infection vs. challenge) and

201 suggest the existence of predictable dynamics.

202

203 We further investigated the microbial community dynamics following infection, and we

204 observed some consistent changes in response to experimental infection type (Fig. 4B). The 205 relative abundance of Tenericutes increased after infection, particularly at day 1 after challenge 
206 (BB25 vs BBB1, student $T$ test $P=0.0114 ; B B 25$ vs BBV1 student $T$ test $P=0.0209$ ). Increase in

207 Verrucomicrobia following allopatric primo-infection (B0/B25 vs BV4, student T test P = 6.53 e-

208 06) and homologous / heterologous challenge (B0/B25 vs BBB4/BBV4, student $T$ test $P=$

209 0.0012) was most prominent at day 4 after infection (Fig. 4B). Interestingly, high relative

210 abundance of Verrucomicrobia in BB25 represents the main difference with naive snail

211 microbial community composition (BO/B25 vs BB25, student $T$ test $P=1.83$ e -05 ) (Fig. 4B). We

212 further observed decrease in Planctomycetes following sympatric primo-infection (B0/B25 vs

213 BB1/BB4, student $T$ test $P=5.7$ e-07) and a decrease in Cyanobacteria following allopatric

214 primo-infection (B0/B25 vs BV1/BV4, student $\mathrm{T}$ test $\mathrm{P}=0.00254)$. Interestingly, the relative

215 abundance of both phyla decreased following the challenge regardless of the challenge type

216 (Planctomycetes B0/B25/BB25 vs BBB1/BBB4/BBV1/BBV4, student $T$ test $P=0.0017$ and

217 Cyanobacteria B0/B25/BB25 vs BBB1/BBB4/BBV1/BBV4, student $T$ test $P=0.000568$ ) (Fig. 4B).

218 Bacteroidetes decreased 25 days after sympatric primo-infection (B0/B25 vs BB25, student T

219 test $\mathrm{P}=0.0467$ ) but increased 4 days after heterologous challenge (BB25 vs BBV4, student T

220 test $P=0.00577)$. To conclude, depending on the phylum, we observed the largest

221 modifications in microbiota composition at day 1 or 4 after primo-infections and challenges.

222 Whereas the microbiota responded differently to sympatric and allopatric primo-infections, we

223 found no significant differences between the homologous or heterologous challenge (see BBB1

224 vs BBV1, Fig. 4) (Table S5).

225

226 Finally, we examined the core microbiota dynamics during infection. Similarly, to the complete

227 microbiota, the core-microbiota, consisting of 62 families, was affected by the type of infection

228 (naive vs primo-infection ( $p=0.003)$ and naive vs challenge $(p=0.003)$ ), by the time of infection 
229 (early ( 1 day) vs late $(4$ days $))(p=0.006)$ and also by the strain of parasite used for primo-

230 infection (SmBRE vs SmVEN) ( $p=0.016$ ) (Table S6). Principal Coordinate Analysis (PCoA) based

231 on Bray-Curtis dissimilarities between the core microbiotas (Fig. S1) yielded very similar results

232 to those based on the entire dataset (Fig. 4A).

233 In addition, we observed that $69.4 \%$ (43) of the core microbiota families were significantly

234 affected by infection. Among those, 6.5\% (4 families) were affected regardless of the infection

235 type (Table S7). Interestingly, these families belong to the most abundant ones (Table S8).

236 Nineteen families (30.6\%) were never affected by infection (Table S7). Those families belong to

237 the less represented ones, with the exception of Xanthomonadaceae, which was the $7^{\text {th }}$ most

238 represented family (see Table S8). Further 32.6\% (14 families) of the core microbiota responded

239 solely to the primo-infection and $4.7 \%$ (2 families) to the challenge (Table S7). Similarly, $20.9 \%$

240 (9 families) of the core microbiota was affected early after infection ( 1 day) and $7 \%$ later ( 3

241 families, day 4). Finally, 23.3\% (10 families) were affected by the SmBRE infection and 9.3\% (4

242 families) by the SmVEN infection (Table S7).

244 Link between the microbiota dysbiosis and B. glabrata antimicrobial immune response

245 The expression level of transcripts encoding antimicrobial peptides and antimicrobial proteins

246 (AMP) was investigated following sympatric or allopatric primo-infection and homologous or

247 heterologous challenges using RNAseq data (Fig. 5 and Fig. S2). Two achacin genes, 5

248 lipopolysaccharide-binding protein / bactericidal permeability-increasing protein (LBP/PBI)

249 genes, and 5 biomphamacin genes were selected based on the Biomphalaria glabrata genome

250 annotation (Adema et al., 2017). In comparison with the naive snails, the LBP/BPI 3.1 and 3.2

251 were over-expressed at day 25 after infection and following the challenges, while all other 
252 genes of this family were under-expressed in all conditions (Fig. S2). The achacins were under-

253 expressed following primo-infection in the sympatric combination only, and at day 25 after

254 primo-infection and following both challenges (Fig. S2). No changes in expression were

255 observed following allopatric primo-infection compared to the naive snails (Fig. S2). Finally, the

256 AMP biomphamacins 1, 4, 5 and 6 were over-expressed throughout the infection stages, except

257 for BV1 and BB25 (Fig.6). The biomphamacin 3 was mainly under-expressed except at BV4 and

258 BBB, where no differential expressions have been detected compared with naive snails (Fig. 5).

259

260 Based on our observations of microbial community shifts, changes in expression of

261 antimicrobial molecules were expected to occur following primo-infections and challenges and

262 not for BB25, where resilience of microbial communities has been observed (Fig. 4 and Fig. S1).

263 In this context, the LBP/BPI and achacin seem not to be linked with dysbiosis, as both remained

264 highly down regulated even at BB25 when communities had already recovered (Fig. S2). All

265 biomphamacins, except the biomphamacin 3, were over-expressed after infection, from day 1

266 in BB infection and day 4 in BV infection and for homologous or heterologous challenges (BBB

267 and BBV). Finally, only a subset of biomphamacins $(1,4,5,6)$ changed in the course of infection

268 but were not differentially expressed at BB25 compared with the naive snails (Fig. 5), 269 suggesting their possible link with dysbiosis.

\section{Discussion}

272 Fine-tuned interactions between microbiota, host immunity and pathogens have been

273 observed in many vertebrate and invertebrate models (Rodrigues et al., 2010; Ramirez et al., 274 2012; Futo et al., 2015; Sansone et al., 2015; Chen et al., 2017). Following an infection by a 
275 pathogen, a host displays an innate or adaptive immune response against the intruder. The

276 activation of such immune response may in turn affect the microbial community structure.

277 Herein, we investigated the interactions between the host immune system, parasite and the

278 bacterial microbiota in an invertebrate model - the gastropod snail Biomphalaria glabrata and

279 its trematode parasite Schistosoma mansoni. Depending on the past evolutionary history

280 between snails and schistosomes, different immune responses against S. mansoni have been

281 observed. We showed recently that in a sympatric interaction, the parasite that coevolved with

282 its host induced a strong immunosuppression, whereas allopatric interaction resulted in a

283 strong host cellular immune response (Portet et al., 2019). Moreover, a cellular immune

284 response was observed following primo-infection, but a humoral immune response was

285 observed following homologous or heterologous challenges (Pinaud et al., 2016). So using

286 appropriate parasite-host combinations, we have the opportunity to modulate the host

287 immune response type and to test changes in bacterial community composition and diversity

288 (dysbiosis). First, studying the global bacterial microbiota community of Biomphalaria snails, we

289 showed that the bacterial alpha diversity was not modified following primo-infection whatever

290 the parasite strain or the time point of infection (Fig. 3, S3 Table). A decrease in alpha diversity

291 is observed exclusively following challenge infections, as reflected by multiple indices (Fig. 3, S3

292 Table). Conversely, primo-infection and challenge strongly affected the bacterial OTU

293 composition (Fig. 4). Moreover, differences in immunobiological interactions

294 (immunosuppression, immune cellular response or immune humoral response) resulted in

295 different microbiota dysbiosis reflected by specific changes in the snail microbial community

296 (Fig. 4). Interestingly, homologous and heterologous challenges activated similar humoral

297 immune response (Pinaud et al., 2016; Portet et al., 2019) that resulted in a similar change of 
298 microbiota alpha diversity and composition (Fig. 3, S3 Table, Fig. 4). Then, four days after

299 infection, regardless of its type, the microbiota was still very different from the one in the naïve

300 snails, but the differences between the primo-infection and challenge disappeared, as apparent

301 from the grouping of BB4, BV4, BBB4 and BBV4 in the PCOA (see Fig. 4). Furthermore, most of

302 the OTUs affected by the primo-infection, returned to their initial state by the day 25 , indicating

303 that the snail microbiota is resilient against the infection (Fig. 4). However, some differences

304 persisted: the Verrucomicrobia phylum remained highly represented in the infected snails at

305 day 25 after primo-infection (Fig. 4B). Interestingly, some Verrucomicrobia species have been

306 recently proposed as a hallmark of healthy gut due to its anti-inflammatory and immune-

307 stimulant properties and its ability to improve gut barrier function (Fujio-Vejar et al., 2017).

308 High abundance of Verrucomicrobia in the recovered snails could thus potentially reflect their

309 role in community restoration (Fig. 4). Moreover, the expansion of a Verrucomicrobia species

310 Akkermansia muciniphilahas been demonstrated in the gut of $S$. mansoni-infected mice,

311 suggesting a potentially functional role of Verrucomicrobia in Schistosoma infection processes

312 in both final and intermediate hosts (Jenkins et al., 2018). We also paid a particular attention to

313 the core-microbiota. Given the various ways to define core microbiota, we considered

314 exclusively the persistent occurrence in the bacterial community in naive Biomphalaria glabrata

315 snails (Astudillo-García et al., 2017). Similarly, to the entire microbiota, the core microbiota was

316 affected by the type of infection (naive vs challenge), by the time of infection (day 1 vs day 4 ) as

317 well as by the parasite strain (SmBRE vs SmVEN) (Table S6). Core microbiota seemed to be

318 affected by immunosuppression or the activation of the immune response in a similar way than

319 the whole microbiota (Fig. S1 and Fig. 4A). Understanding the shifts in core microbiota

320 following infection is important as the long-term stability and persistent occurrence of 
321 beneficial microbes and their associated functions may contribute to host health and well-being

322 to maintain host functionality and fitness toward changing ecological environment or

323 environmental stress (Nyholm and McFall-Ngai, 2004; Lozupone et al., 2012; McFall-Ngai et al., 324 2013).

325 Given that infections affect the total and core microbiota structure, we explored the 326 antimicrobial immune response following infection to find potential molecular mechanisms 327 involved in the observed microbiota dysbiosis (Fig. 5 and Fig. S2). We analysed antimicrobial 328 peptides (AMP) and antimicrobial proteins and our results suggest that only the AMP belonging to biomphamacin family may potentially affect the microbial communities in response to 330 Schistosoma infection. Indeed, even though antimicrobial families can be involved in complex 331 process of regulation or of control of microbiota communities, solely the biomphamacins AMP 332 family members specifically displayed an expression pattern that can be linked with the 333 observed dysbiosis of Biomphalaria bacterial communities (Fig. 5). Even if AMPs is often 334 considered as the main immune pathway responsible for microbiota regulation (Onchuru and 335 Kaltenpoth, 2019), an overall modification of the immune system may also be considered as a 336 cause of dysbiosis. The potential role of immune recognition or ROS/NOS pathways as to be 337 considered (Wang et al., 2014; Budachetri and Karim, 2015; Yang et al., 2015; Yang et al., 2016) 338 and this will deserve further investigations in the present biological experimental model.

339 It has been shown that the immune system is a key determinant of host-associated bacterial 340 communities in many biological systems. Two mechanisms have been proposed to explain host341 microbiota interactions through crosstalk with the host innate immune system. The first one 342 proposes that the host immune system exerts constant pressure on the microbiota in order to 343 maintain homeostasis (Hooper et al., 2012), the host immune system can thus control the 
344 composition of the resident microbiota (Zhang et al., 2015). According to this hypothesis, any

345 changes in host immune response to infection would potentially affect the resident microbiota

346 diversity and composition. The second mechanism proposes that the immune system would be

347 tolerant to weak and continuous antigenic immune stimulations experienced during a lifespan

348 (Pradeu and Eric, 2014), and thus that host immune system would not exert any pressure on

349 the resident microbiota. In this case, any changes in host immune response to infection would

350 potentially not affect the resident microbiota diversity and composition.

351 Interestingly, numerous studies demonstrate a direct control of microbial communities by the

352 host immune system. As an example, species-specific antimicrobial peptides can shape species-

353 specific bacterial associations in Hydra (Franzenburg et al., 2013). Other immune pathways

354 have been also demonstrated to regulate or control the microbiota communities, like the

355 intestinal homeo-box gene Caudal in Drosophila (Ryu et al., 2008), or neuropeptides with

356 antibacterial activity which are secreted to shape the microbiome on the body surface of Hydra

357 (Augustin et al., 2017), even host lectins were demonstrated to stabilize microbiota

358 communities (Dinh et al., 2018).

359 Thus, if biotic stress (i.e. an infection) modifies the expression of antimicrobial peptides or

360 other immune related pathways, an effect on the microbial communities can be expected. Our

361 results indicate that bacterial communities could indeed be shaped by the immune system of $B$.

362 glabrata. Based on the hypothesis proposed by Hooper and collaborators (Hooper and

363 Macpherson, 2010; Hooper et al., 2012; Dinh et al., 2018), it seems that the immune system of

364 B. glabrata snails maintains or controls the microbial communities permanently. Therefore,

365 following an infection, the immune system is diverted from its function of managing the

366 microbiota and consequently the bacterial communities evade its regulation, resulting in 
367 changes of their composition and diversity. When the immune response returns to a basal level,

368 the microbiota is again under-control and the microbiota shows its resilience by returning to its

369 "healthy" state (Hooper and Macpherson, 2010; Hooper et al., 2012), as observed in the

370 present study (Fig. 4). In other words, the immune system is likely no longer able to maintain

371 the microbiota homeostasis after infection (resulting in dysbiosis), which, may in turn affect

372 host homeostasis or fitness (Contijoch et al., 2019; Warne et al., 2019) (Contijoch, Britton et al.

373 2019, Warne, Kirschman et al. 2019).

374 Changes in microbiota composition may result from shifts in the abundance of specific bacterial

375 groups participating in anti-pathogen response, or just be a collateral effect of the immune

376 response activation against metazoan parasite infection. This question will deserve further

377 investigation, by testing S. mansoni prevalence and intensity in experimental infections of

378 Biomphalaria glabrata snails following antibiotic treatment or microbiota transplantation.

379

380

381

382

383

384

385

386

387

388

389
In order to fully understand Schistosomiasis transmission and to develop new ways to control the expansion of this widespread human parasitic disease in the field, it will be crucial to determine if the snail-associated bacterial communities affect the parasite transmission. For example, expanding knowledge on Biomphalaria snail microbiota is an essential step for developing paratransgenetic solutions to the spread of Schistosomiasis, involving the use of transgenic bacteria expressing foreign gene products (i.e., schistosomicidal compounds) that can reduce host competence and block pathogen development or transmission when introduced into the microbiota of vector snail field populations (Aksoy et al., 2008; CoutinhoAbreu et al., 2010; Gilbert et al., 2016). 


\section{Experimental procedures}

\section{Ethical statements}

392 Our laboratory holds permit \# A66040 for experiments on animals, which was obtained from

393 the French Ministry of Agriculture and Fisheries and the French Ministry of National Education,

394 Research, and Technology. The housing, breeding and care of the utilized animals followed the 395 ethical requirements of our country. The experimenter possesses an official certificate for

396 animal experimentation from both of the above-listed French ministries (Decree \# 87-848,

397 October 19, 1987). The various protocols used in this study have been approved by the French 398 veterinary agency of the DRAAF Languedoc-Roussillon (Direction Régionale de I'Alimentation, 399 de l'Agriculture et de la Forêt), Montpellier, France (authorization \# 007083).

Biological material

402 In this study, we used a single strain of the host snail, the albino Brazilian strain of Biomphalaria 403 glabrata (BgBRE) and two strains of the trematode parasite Schistosoma mansoni: a Brazilian 404 strain (SmBRE, for sympatric infection) and a Venezuelan strain (SmVEN, for allopatric 405 infection). BgBRE and SmBRE strains originate from the locality of Recife, Brazil; the SmVEN 406 parasite strain was recovered from the locality of Guacara, Venezuela. All host and parasite 407 strains were maintained in the laboratory on their respective sympatric snail hosts (SmBRE on 408 BgBRE and SmVEN on BgVEN). The snails were reared at constant temperature of $26^{\circ} \mathrm{C}$ and fed 409 only with washed salad every 3 days.

410

411 Experimental Infections (Fig. 1)

412 In order to decipher the inter-individual structure of snail microbiota as well as investigate the 
413 influence of different snail immune stimulations on its microbiota structure and dynamics, we

414 applied a two-step experimental infection protocol (Fig. 1).

415 Briefly, BgBRE snails were primo-infected with one of the two parasite strains (SmBRE

416 sympatric or SmVEN allopatric). The snails were then sampled 1 and 4 days following primo-

417 infection by both parasite strains and 25 days after primo-infection for SmBRE infection

418 condition. Then, the BgBRE snails primo-infected with SmBRE were challenged (25 days after

419 the primo-infection) with SmBRE or SmVEN parasite strains for homologous or heterologous

420 challenges respectively. The challenged snails were then sampled 1 and 4 days following

421 challenge (Fig. 1). For all experimental infections, the snails were individually exposed for $12 \mathrm{~h}$

422 to 10 miracidia in $5 \mathrm{~mL}$ of pond water, thereafter snails were returned to the water tanks and 423 separated according to the parasite strain and infection conditions.

We performed a primo-infection of 63 BgBRE snails with either SmBRE (sympatric, 49 snails) or

427 SmVEN (allopatric, 14 snails) strains. Then, 25 days after the primo-infection, we challenged a 428 subset (28 snails) of SmBRE-primo-infected snails with either SmBRE (14 snails) (homologous challenge) or SmVEN (14 snails) (heterologous challenge).

430 To examine the effect of primo-infection on the snail microbiota, we sampled 7 whole snails for 431 each infection combination on day one (named BB1 and BV1; the first letter refers to the 432 Biomphalaria strain BgBRE and the second letter refers to the origin of S. mansoni strains used 433 for primo-infection, SmBRE or SmVEN), on day four (BB4 and BV4), and on day twenty-five 434 (BB25, no SmVEN-infected snails were sampled on day 25) after the primo-infection. This BB25 sample is used as control for microbiota changes observed following challenge infections. Thus 
436 then, we sampled 7 whole-snails on day one after the challenge (BBB1 and BBV1; the third

437 letter refers to the origin of the S. mansoni strain used for the challenge, SmBRE or SmVEN) and 438 on day four (BBB4 and BBV4) after the challenge. In addition, we used 6 naive snails collected at 439 the beginning of the experiment (BO) and 6 naive snails collected at the time of challenge (i.e. 440 day 25 , named B25) as controls to assess the variability and stability of the BgBRE snail's 441 bacterial communities in our breeding and rearing laboratory conditions. Snails were not fed 24 442 hours before the DNA extraction for metabarcoding analysis. $\underline{\text { Infection and sampling for host antimicrobial immune response }}$ We performed a primo-infection of 180 BgBRE snails with either SmBRE (sympatric, 140 snails) or SmVEN (allopatric, 40 snails). Then, 25 days after infection, we challenged a subset (80 snails) of SmBRE-infected snails with either SmBRE (40 snails) (homologous challenge) or 448 SmVEN (40 snails) (heterologous challenge) (Portet et al., 2019).

449 To study the primo-infection transcriptomic immune response of the snail, we took 20 snails 450 pooled for each infection type on day one (BB1 and BV1), four (BB4 and BV4), and twenty-five 451 (BB25). After the challenge with SmBRE or SmVEN, we sampled 20 snails in each condition at 452 days 1 and 4 . Then samples of day 1 and day 4 were mixed together into a single sample referring to $\mathrm{BBB}$ or BBV. In addition, we used 2 pools of 30 naive snails (BO.1 and B0.2) to establish the basal snail transcriptomic activity (Portet et al., 2019). 
Immediately after sampling, snail shells were cleaned with alcohol and removed, whole snails

were then frozen in liquid nitrogen and grounded. The total DNA was extracted with DNeasy

460 Blood and Tissue Kit (Qiagen) according to the manufacturer's protocol. The DNA quantification

461 was performed by Qubit 2.0 Fluorometer, using dsRNA BR Assay kit. Individual 16S rDNA

462 amplicon libraries were generated using the 341F (CCTACGGGNGGCWGCAG) and 805R

463 (GACTACHVGGGTATCTAATCC) primers targeting the variable V3-V4 loops (Klindworth et al.,

464 2013). Paired-end sequencing with 250 bp read length was performed on the Illumina MiSeq

465 sequencing system (Genome Québec, Montréal, Québec, Canada) using the v2 chemistry

466 according to the manufacturer's protocol.

467

RNA extraction and transcriptomic sequencing

469 Immediately after sampling, snail shells were cleaned with alcohol and removed, then snails

470 were pooled according to infection type. Total RNA was extracted using TRIZOL (Sigma Life

471 Science, USA) according to the manufacturer's instructions. For BBB and BBV equimolar

472 amounts of RNA extracted from molluscs challenged at both 1 and 4 days were mixed together

473 into a single sample. mRNAs were sequenced in paired-end 72-bp read lengths, using the

474 mRNA-Seq kit for transcriptome sequencing on Illumina Genome Analyzer II (MGX-Montpellier

475 GenomiX, Montpellier, France).

476

477 Microbiota analysis

$478 \quad$ Data analysis of 165 sequences

479 The FROGS pipeline (Find Rapidly OTU with Galaxy Solution) implemented on a galaxy instance 480 (http://sigenae-workbench.toulouse.inra.fr/galaxy/) was used for data processing (Escudié et 
481 al., 2017). Briefly, paired reads were merged using FLASH (Magoc and Salzberg, 2011). After

482 denoising and primer/adapters removal (Didion et al., 2017), de novo clustering was done using

483 SWARM, which uses a local clustering threshold, with aggregation distance $d=3$ after denoising.

484 Chimeras were removed using VSEARCH (Rognes et al., 2016). We filtered out the singletons 485 and performed taxonomic assignment using Blast+ against the Silva database (release 128).

486 All statistical analyses were done using R v3.3.1 ( $R$ : a language and environment for 487 statistical computing, 2008; R Development Core Team, R Foundation for Statistical Computing, 488 Vienna, Austria [http://www.R-project.org]). We used the phyloseq R package for community composition analysis (McMurdie and Holmes, 2013) to infer alpha diversity metrics as well as

490 beta diversity (between sample distance). Beta diversity was examined by Principal Coordinate 491 Analysis (PCoA) using the Bray-Curtis distance matrices. We performed a Mann Whitney $U$ test $492(\mathrm{p}<0.05)$ to compare alpha diversity and one-way PERMANOVA with a Benjamini \& Hochberg 493 post-hoc to analyze beta diversity between the experimental groups. For all analyses, the 494 threshold significance level was set at 0.05 . Analysis of core-microbiota

496 We defined the core-microbiota as the set of bacterial families that were present in $100 \%$ of 497 the naive individuals excluding unknown or multi-affiliations at lower taxonomic ranks (Table 498 S1). We then used the abundances of OTUs belonging to these families to examine the 499 composition of the core-microbiota.

500 To check if the core-microbiota is affected by infection, we compared the abundance of core 501 families between infected snails and naive conditions with a one-way PERMANOVA with a 502 Benjamini \& Hochberg post-hoc correction. Moreover, a frequency test was performed to 503 determine which specific families were affected during infection. The number of significantly 
504 differentially represented families at each sampling day (1, 4 and 25 days after primo infections

505 and 1, 4 days after challenges) was calculated to assess the temporal variability during the 506 course of infection.

507

508 Transcriptome analysis of antimicrobial immune response

$509 \quad$ Antimicrobial response

510 An antimicrobial transcriptome was built from transcripts known to be involved in Biomphalaria

511 immune response against bacteria (antimicrobial peptides: biomphamacin or antimicrobial

512 proteins: LBP/BPI and achacin; see Table S2 for details). The full-length sequences of these

513 transcripts were recovered from GenBank and the Biomphalaria genome (Adema et al., 2017)

514 and joined in a subset that represents the antimicrobial transcriptome of $B$. glabrata. This

515 antimicrobial transcriptome was then concatenated with a de-novo assembled transcriptome

516 of Biomphalaria available in our laboratory (see (Dheilly et al., 2015; Pinaud et al., 2016;

517 Galinier et al., 2017) for details) and uploaded on the Galaxy server. Before concatenation, a

518 blastn (70\% identity and $90 \%$ coverage) was conducted to identify redundant transcripts across

519 the transcriptomes. Redundant transcripts were then discarded using CDhitEst to avoid

520 mapping errors and bias in read counts when using Bowtie2 package.

$521 \quad$ Differential expression analysis

522 High-quality reads (Phred score $>29$ ) were aligned to the concatenated transcriptome using

523 Bowtie2 (v.2.0.2), which was run locally on a Galaxy server. The DESeq2 (v2.12) R package was

524 used to identify differential expression levels between uninfected (B0.1 and B0.2) and infected

525 conditions ( $p$ value $<0.05$ ).

526 


\section{Acknowledgments}

528 BG was supported by ANR JCJC INVIMORY (number ANR 13-JSV7-0009) from the French

529 National Research Agency (ANR). The authors want to thanks the DHOF program of the

530 UMR5244/IHPE (http://ihpe.univ-perp.fr/en/ihpe-transversal-holobiont/) for partial financial

531 support. This study is set within the framework of the "Laboratoires d'Excellences (LABEX)"

532 TULIP (ANR-10-LABX-41).

533

534 Reference

535 Adema, C.M., Hillier, L.W., Jones, C.S., Loker, E.S., Knight, M., Minx, P. et al. (2017) Whole

536 genome analysis of a schistosomiasis-transmitting freshwater snail. Nature communications 8 :

53715451.

538 Aksoy, S., Weiss, B., and Attardo, G. (2008) Paratransgenesis applied for control of tsetse

539 transmitted sleeping sickness. Adv Exp Med Biol 627: 35-48.

540 Allan, E.R.O., Tennessen, J.A., Sharpton, T.J., and Blouin, M.S. (2018) Allelic Variation in a Single

541 Genomic Region Alters the Microbiome of the Snail Biomphalaria glabrata. J Hered 109: 604-

542609.

543 Astudillo-García, C., Bell James, J., Webster Nicole, S., Glasl, B., Jompa, J., Montoya Jose, M.,

544 and Taylor Michael, W. (2017) Evaluating the core microbiota in complex communities: A

545 systematic investigation. Environmental Microbiology 19: 1450-1462.

546 Augustin, R., Fraune, S., and Bosch, T.C.G. (2010) How Hydra senses and destroys microbes.

547 Seminars in Immunology 22: 54-58.

548 Augustin, R., Schröder, K., Murillo Rincón, A.P., Fraune, S., Anton-Erxleben, F., Herbst, E.-M. et

549 al. (2017) A secreted antibacterial neuropeptide shapes the microbiome of Hydra. Nature

550 communications 8: 698. 
551 Bosch, T.C. (2013) Cnidarian-microbe interactions and the origin of innate immunity in

552 metazoans. Annu Rev Microbiol 67: 499-518.

553 Bouchut, A., Roger, E., Gourbal, B., Grunau, C., Coustau, C., and Mitta, G. (2008) The

554 compatibility polymorphism in invertebrate host/trematode interactions: research of molecular

555 determinants. Parasite 15: 304-309.

556 Budachetri, K., and Karim, S. (2015) An insight into the functional role of thioredoxin reductase,

557 a selenoprotein, in maintaining normal native microbiota in the Gulf Coast tick (Amblyomma

558 maculatum). Insect Mol Biol 24: 570-581.

559 Chen, F., Krasity, B.C., Peyer, S.M., Koehler, S., Ruby, E.G., Zhang, X., and McFall-Ngai, M.J.

560 (2017) Bactericidal Permeability-Increasing Proteins Shape Host-Microbe Interactions. mBio 8:

561 e00040-00017.

562 Cirimotich, C.M., Dong, Y., Clayton, A.M., Sandiford, S.L., Souza-Neto, J.A., Mulenga, M., and

563 Dimopoulos, G. (2011) Natural microbe - mediated refractoriness to Plasmodium infection in

564 Anopheles gambiae. Science 332: 855-858.

565 Contijoch, E.J., Britton, G.J., Yang, C., Mogno, I., Li, Z., Ng, R. et al. (2019) Gut microbiota density

566 influences host physiology and is shaped by host and microbial factors. Elife 8: pii: e40553.

567 Coustau, C., Gourbal, B., Duval, D., Yoshino, T.P., Adema, C.M., and Mitta, G. (2015) Advances in

568 gastropod immunity from the study of the interaction between the snail Biomphalaria glabrata

569 and its parasites: A review of research progress over the last decade. Fish \& Shellfish

570 Immunology 46: 5-16.

571 Coutinho-Abreu, I.V., Zhu, K.Y., and Ramalho-Ortigao, M. (2010) Transgenesis and

572 paratransgenesis to control insect-borne diseases: current status and future challenges.

573 Parasitol Int 59: 1-8. 
574 Dheilly, N.M., Duval, D., Mouahid, G., Emans, R., Allienne, J.-F., Galinier, R. et al. (2015) A family

575 of variable immunoglobulin and lectin domain containing molecules in the snail Biomphalaria

576 glabrata. Developmental \& Comparative Immunology 48: 234-243.

577 Didion, J.P., Martin, M., and Collins, F.S. (2017) Atropos: specific, sensitive, and speedy

578 trimming of sequencing reads. PeerJ 5: e3720.

579 Dinh, C., Farinholt, T., Hirose, S., Zhuchenko, O., and Kuspa, A. (2018) Lectins modulate the

580 microbiota of social amoebae. Science 361: 402-406.

581 Doenhoff, M.J., Hagan, P., Cioli, D., Southgate, V., Pica-Mattoccia, L., Botros, S. et al. (2009)

582 Praziquantel: its use in control of schistosomiasis in sub-Saharan Africa and current research

583 needs. Parasitology 136: 1825-1835.

584 Ducklow, H.W., Boyle, P.J., Maugel, P.W., Strong, C., and Mitchell, R. (1979) Bacterial flora of

585 the schistosome vector snail Biomphalaria glabrata. Applied and Environmental Microbiology

586 38: $667-672$.

587 Escudié, F., Auer, L., Bernard, M., Mariadassou, M., Cauquil, L., Vidal, K. et al. (2017) FROGS:

588 Find, Rapidly, OTUs with Galaxy Solution. Bioinformatics: btx791.

589 Fallon, P.G., and Doenhoff, M.J. (1994) Drug-Resistant Schistosomiasis: Resistance to

590 Praziquantel and Oxamniquine Induced in Schistosoma Mansoni in Mice is Drug Specific. The

591 American Journal of Tropical Medicine and Hygiene 51: 83-88.

592 Franzenburg, S., Fraune, S., Künzel, S., Baines, J.F., Domazet-Lošo, T., and Bosch, T.C.G. (2012)

593 MyD88-deficient Hydra reveal an ancient function of TLR signaling in sensing bacterial

594 colonizers. Proceedings of the National Academy of Sciences of the United States of America

595 109: 19374-19379. 
596 Franzenburg, S., Walter, J., Künzel, S., Wang, J., Baines, J.F., Bosch, T.C.G., and Fraune, S. (2013)

597 Distinct antimicrobial peptide expression determines host species-specific bacterial

598 associations. Proceedings of the National Academy of Sciences of the United States of America

599 110: E3730-E3738.

600 Fraune, S., Augustin, R., Anton-Erxleben, F., Wittlieb, J., Gelhaus, C., Klimovich, V.B. et al. (2010)

601 In an early branching metazoan, bacterial colonization of the embryo is controlled by maternal

602 antimicrobial peptides. Proceedings of the National Academy of Sciences of the United States of

603 America 107: 18067-18072.

604 Fujio-Vejar, S., Vasquez, Y., Morales, P., Magne, F., Vera-Wolf, P., Ugalde, J.A. et al. (2017) The

605 Gut Microbiota of Healthy Chilean Subjects Reveals a High Abundance of the Phylum

606 Verrucomicrobia. Front Microbio/ 8: 1221.

607 Futo, M., Armitage, S.A., and Kurtz, J. (2015) Microbiota Plays a Role in Oral Immune Priming in

608 Tribolium castaneum. Front Microbiol 6: 1383.

609 Galinier, R., Roger, E., Moné, Y., Duval, D., Portet, A., Pinaud, S. et al. (2017) A multistrain

610 approach to studying the mechanisms underlying compatibility in the interaction between

611 Biomphalaria glabrata and Schistosoma mansoni. PLOS Neglected Tropical Diseases 11:

612 e0005398.

613 Gendrin, M. (2017) A Swiss Army Knife to Cut Malaria Transmission. Cell Host \& Microbe 22:

$614 \quad 577-579$.

615 Gilbert, J.A., Medlock, J., Townsend, J.P., Aksoy, S., Ndeffo Mbah, M., and Galvani, A.P. (2016)

616 Determinants of Human African Trypanosomiasis Elimination via Paratransgenesis. PLoS Negl

617 Trop Dis 10: e0004465. 
618 Hooper, L.V., and Macpherson, A.J. (2010) Immune adaptations that maintain homeostasis with

619 the intestinal microbiota. Nat Rev Immunol 10: 159-169.

620 Hooper, L.V., Littman, D.R., and Macpherson, A.J. (2012) Interactions between the microbiota

621 and the immune system. Science (New York, N.Y.) 336: 1268-1273.

622 Jenkins, T.P., Peachey, L.E., Ajami, N.J., MacDonald, A.S., Hsieh, M.H., Brindley, P.J. et al. (2018)

623 Schistosoma mansoni infection is associated with quantitative and qualitative modifications of

624 the mammalian intestinal microbiota. Sci Rep 8: 12072.

625 Joshi, D., Pan, X., McFadden, M.J., Bevins, D., Liang, X., Lu, P. et al. (2017) The Maternally

626 Inheritable Wolbachia wAlbB Induces Refractoriness to Plasmodium berghei in Anopheles

627 stephensi. Frontiers in Microbiology 8: 366.

628 Klindworth, A., Pruesse, E., Schweer, T., Peplies, J., Quast, C., Horn, M., and Glockner, F.O.

629 (2013) Evaluation of general 16S ribosomal RNA gene PCR primers for classical and next-

630 generation sequencing-based diversity studies. Nucleic Acids Res 41: e1.

631 Lozupone, C.A., Stombaugh, J.I., Gordon, J.I., Jansson, J.K., and Knight, R. (2012) Diversity,

632 stability and resilience of the human gut microbiota. Nature 489: 220-230.

633 Magoc, T., and Salzberg, S.L. (2011) FLASH: fast length adjustment of short reads to improve

634 genome assemblies. Bioinformatics 27: 2957-2963.

635 McFall-Ngai, M., Hadfield, M.G., Bosch, T.C., Carey, H.V., Domazet-Loso, T., Douglas, A.E. et al.

636 (2013) Animals in a bacterial world, a new imperative for the life sciences. Proc Natl Acad SciU

637 SA 110: 3229-3236.

638 McMurdie, P.J., and Holmes, S. (2013) phyloseq: an R package for reproducible interactive

639 analysis and graphics of microbiome census data. PLoS One 8: e61217. 
640 Mitta, G., Adema, C.M., Gourbal, B., Loker, E.S., and Theron, A. (2012) Compatibility

641 polymorphism in snail/schistosome interactions: From field to theory to molecular

642 mechanisms. Dev Comp Immunol 37: 1-8.

643 Mitta, G., Gourbal, B., Grunau, C., Knight, M., Bridger, J.M., and Théron, A. (2017) Chapter

644 Three - The Compatibility Between Biomphalaria glabrata Snails and Schistosoma mansoni: An

645 Increasingly Complex Puzzle. Advances in Parasitology 97: 111-145.

646 Mitta, G., Galinier, R., Tisseyre, P., Allienne, J.F., Girerd-Chambaz, Y., Guillou, F. et al. (2005)

647 Gene discovery and expression analysis of immune-relevant genes from Biomphalaria glabrata

648 hemocytes. Developmental and comparative immunology 29: 393-407.

649 Mone, Y., Gourbal, B., Duval, D., Du Pasquier, L., Kieffer-Jaquinod, S., and Mitta, G. (2010) A

650 large repertoire of parasite epitopes matched by a large repertoire of host immune receptors in

651 an invertebrate host/parasite model. PLoS Negl Trop Dis 4: pii: e813.

652 Moné, Y., Ribou, A.-C., Cosseau, C., Duval, D., Théron, A., Mitta, G., and Gourbal, B. (2011) An

653 example of molecular co-evolution: Reactive oxygen species (ROS) and ROS scavenger levels in

654 Schistosoma mansoni/Biomphalaria glabrata interactions. International Journal for Parasitology

$655 \quad 41: 721-730$.

656 Narasimhan, S., Schuijt, T.J., Abraham, N.M., Rajeevan, N., Coumou, J., Graham, M. et al. (2017)

657 Modulation of the tick gut milieu by a secreted tick protein favors Borrelia burgdorferi

658 colonization. Nature communications 8: 184.

659 Nyholm, S.V., and McFall-Ngai, M.J. (2004) The winnowing: establishing the squid-vibrio

660 symbiosis. Nat Rev Microbiol 2: 632-642.

661 Onchuru, T.O., and Kaltenpoth, M. (2019) Established cotton stainer gut bacterial mutualists

662 evade regulation by host antimicrobial peptides. Appl Environ Microbiol pii: AEM.00738-19. . 
663 Pinaud, S., Portela, J., Duval, D., Nowacki, F.C., Olive, M.-A., Allienne, J.-F. et al. (2016) A Shift

664 from Cellular to Humoral Responses Contributes to Innate Immune Memory in the Vector Snail

665 Biomphalaria glabrata. PLOS Pathogens 12: e1005361.

666 Portet, A., Pinaud, S., Chaparro, C., Galinier, R., Dheilly, N.M., Portela, J. et al. (2019) Sympatric

667 versus allopatric evolutionary contexts shape differential immune response in Biomphalaria /

668 Schistosoma interaction. PLoS Pathog 15: e1007647.

669 Pradeu, T., and Eric, V. (2014) The discontinuity theory of immunity. Sci Immunol 1: 1-9.

670 Ramirez, J.L., Souza-Neto, J., Cosme, R.T., Rovira, J., Ortiz, A., Pascale, J.M., and Dimopoulos, G.

671 (2012) Reciprocal tripartite interactions between the Aedes aegypti midgut microbiota, innate

672 immune system and dengue virus influences vector competence. PLOS Neglected Tropical

673 Diseases 6: 1-11.

674 Rodrigues, J., Brayner, F.A., Alves, L.C., Dixit, R., and Barillas-mury, C. (2010) Hemocyte

675 Differentiation Mediates Innate Immune Memory in Anopheles gambiae Mosquitoes. Science

676 329: 1353-1355.

677 Rognes, T., Flouri, T., Nichols, B., Quince, C., and Mahe, F. (2016) VSEARCH: a versatile open

678 source tool for metagenomics. PeerJ 4: e2584.

679 Ryu, J.-H., Kim, S.-H., Lee, H.-Y., Bai, J.Y., Nam, Y.-D., Bae, J.-W. et al. (2008) Innate Immune

680 Homeostasis by the Homeobox Gene \&lt;em\&gt;Caudal\&It;/em\&gt; and Commensal-Gut

681 Mutualism in \&lt;em\&gt;Drosophila\&lt;/em\&amp;gt. Science 319: 777.

682 Sansone, C.L., Cohen, J., Yasunaga, A., Xu, J., Osborn, G., Subramanian, H. et al. (2015)

683 Microbiota-dependent priming of antiviral intestinal immunity in Drosophila. Cell Host and

684 Microbe 18: 571-581. 
686 (2013) Characterization of the bacterial microbiota of Biomphalaria glabrata (Say, 1818)

687 (Mollusca: Gastropoda) from Brazil. Letters in Applied Microbiology 57: 19-25.

688 Tennessen, J.A., Théron, A., Marine, M., Yeh, J.-Y., Rognon, A., and Blouin, M.S. (2015)

689 Hyperdiverse Gene Cluster in Snail Host Conveys Resistance to Human Schistosome Parasites.

$690 \quad$ PLoS Genetics 11: e1005067.

691 Wang, X.W., Xu, J.D., Zhao, X.F., Vasta, G.R., and Wang, J.X. (2014) A shrimp C-type lectin

692 inhibits proliferation of the hemolymph microbiota by maintaining the expression of

693 antimicrobial peptides. J Biol Chem 289: 11779-11790.

694 Warne, R.W., Kirschman, L., and Zeglin, L. (2019) Manipulation of gut microbiota during critical

695 developmental windows affects host physiological performance and disease susceptibility

696 across ontogeny. J Anim Ecol Epub ahead of print.

697 WHO (2002) TDR Strategic Direction for Research: Schistosomiasis. World Health Organization

698 Information.

699 Xi, Z., Ramirez, J.L., and Dimopoulos, G. (2008) The Aedes aegypti toll pathway controls dengue

$700 \quad$ virus infection. PLOS Pathogens 4(7):e1000098.

701 Yang, H.T., Yang, M.C., Sun, J.J., Shi, X.Z., Zhao, X.F., and Wang, J.X. (2016) Dual oxidases

702 participate in the regulation of intestinal microbiotic homeostasis in the kuruma shrimp

703 Marsupenaeus japonicus. Dev Comp Immunol 59: 153-163.

704 Yang, H.T., Yang, M.C., Sun, J.J., Guo, F., Lan, J.F., Wang, X.W. et al. (2015) Catalase eliminates

705 reactive oxygen species and influences the intestinal microbiota of shrimp. Fish Shellfish

706 Immunol 47: 63-73. 
bioRxiv preprint doi: https://doi.org/10.1101/386623; this version posted June 4, 2019. The copyright holder for this preprint (which was not certified by peer review) is the author/funder. All rights reserved. No reuse allowed without permission.

707 Zhang, H., Sparks, J.B., Karyala, S.V., Settlage, R., and Luo, X.M. (2015) Host adaptive immunity

708 alters gut microbiota. ISME J 9: 770-781.

709

710

711

712

713 


\section{Legends to Figures}

715

716 Fig. 1: Experimental protocol

717

\section{Fig. 2: Biomphalaria glabrata microbiota characterization}

719 Characterisation of the Biomphalaria bacterial microbiota of six naive snails recovered at the

720 start of experimentation (B0.1; B0.2; BO.3; BO.4; B0.5 and BO.6) and 6 naive snails recovered 25

721 days after the start of experimentation (B25.1; B25.2; B25.3; B25.4; B25.5 and B25.6). A.

722 Phylum level composition of the 20 most abundant OTUs phyla among the 12 naive snails. B.

723 The Venn diagram represents the number of the 97 OTUs families which shared between the 6

724 naive snails at BO (left Venn diagram), and between the 6 naive snails at B25 (right Venn 725 diagram).

726

727 Fig. 3: Microbiota alpha Diversity

728 Boxplots of Chao1 and Shannon indices for all samples. For the Naive condition, B0 and B25

729 snails were pooled; BB: primo infection of BgBRE by SmBRE; BV: primo infection of BgBRE by

730 SmVEN; BBB: primo infection of BgBRE by SmBRE and challenge by SmBRE; BBV: primo

731 infection of BgBRE by SmBRE and challenge by SmVEN. The time point is mentioned with 1, 4 or

73225 corresponding to the day after primo-infection or challenge. The differences between naive 733 and infected conditions were tested with a Mann-Whitney $U$ test and significant differences 734 mentioned with “*”. 
737 Dynamics of the bacterial microbiota of Biomphalaria glabrata following Schistosoma primo-

738 infection and challenge. A. Functional diversity comparisons of Biomphalaria microbiota along

739 infection. Principal coordinate analysis of pairwise Bray-Curtis distances between all infection

740 type and time samples. Axes represent the two synthetic variables explaining the greatest

741 proportion of variation in the data set. The sample name indicated in the figure corresponds to

742 the centroid of all the biological replicates points of the respective experimental sample. B.

743 Phylum level composition of the 20 most abundant OTUs among all points of the kinetic. In this

744 representation, the replicate naive snails were pooled for more readability.

745

746 Fig. 5: Differential gene expression of Biomphamacin antimicrobial peptides

747 Log2FC (fold change) of antimicrobial immune transcripts between naive and infected snails

748 inferred from previous RNAseq analysis on the same experiment. A positive Log2 fold-change

749 indicates over-expression in infected snails compared to the naive snails. Antimicrobial peptide

750 families included 6 Biomphamacins (macin-like AMPs) consisting of 6 genes (shade of green).

751

752 Fig. S1: Core Microbiota analysis

753 Modifications of the bacterial core microbiota of Biomphalaria glabrata following Schistosoma

754 primo-infection and challenge. After a one-way Permanova with a Benjamini \& Hochberg post-

755 hoc, a frequency test was realised to determine which specific families were affected during

756 infection. A. Proportion of OTU Families affected by infection are represented by colour circles.

757 The Venn diagram shows more precisely the specific impact of infections on the OTU families

758 partially influenced. B. Heatmap corresponding to all core microbiota with clustering on Familiy 
759 modification patterns between conditions. The scale of red correspond to ratio between naive

760 condition and each point of infection.

761

762 Fig. S2: Differential gene expression of LBP/BPI and Achacin antimicrobial protein families

763 Log2FC (fold change) of antimicrobial immune transcripts between naive and infected snails

764 inferred from previous RNAseq analysis on the same experiment. A positive Log2 fold-change

765 indicates over-expression in infected snails compared to the naive snails. Antimicrobial protein

766 families: LBP/BPIs (Lipopolysaccharide-binding protein / Bactericidal Permeability-Increasing

767 protein) consisting of 5 genes (in blue) and achacins consisting of 2 genes (in orange).

768

769

770

771 


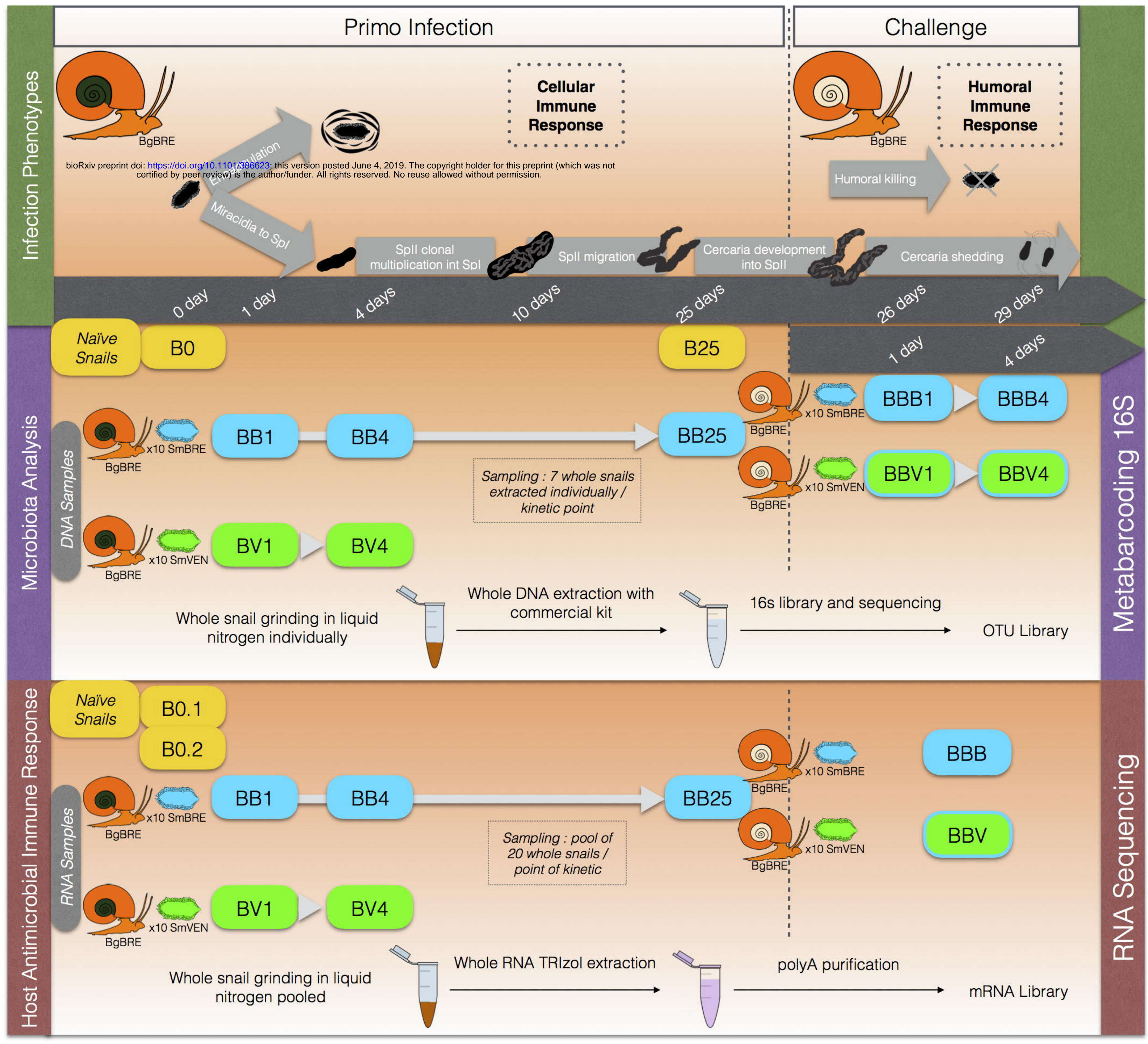



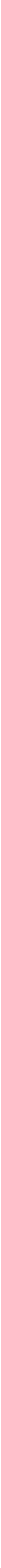


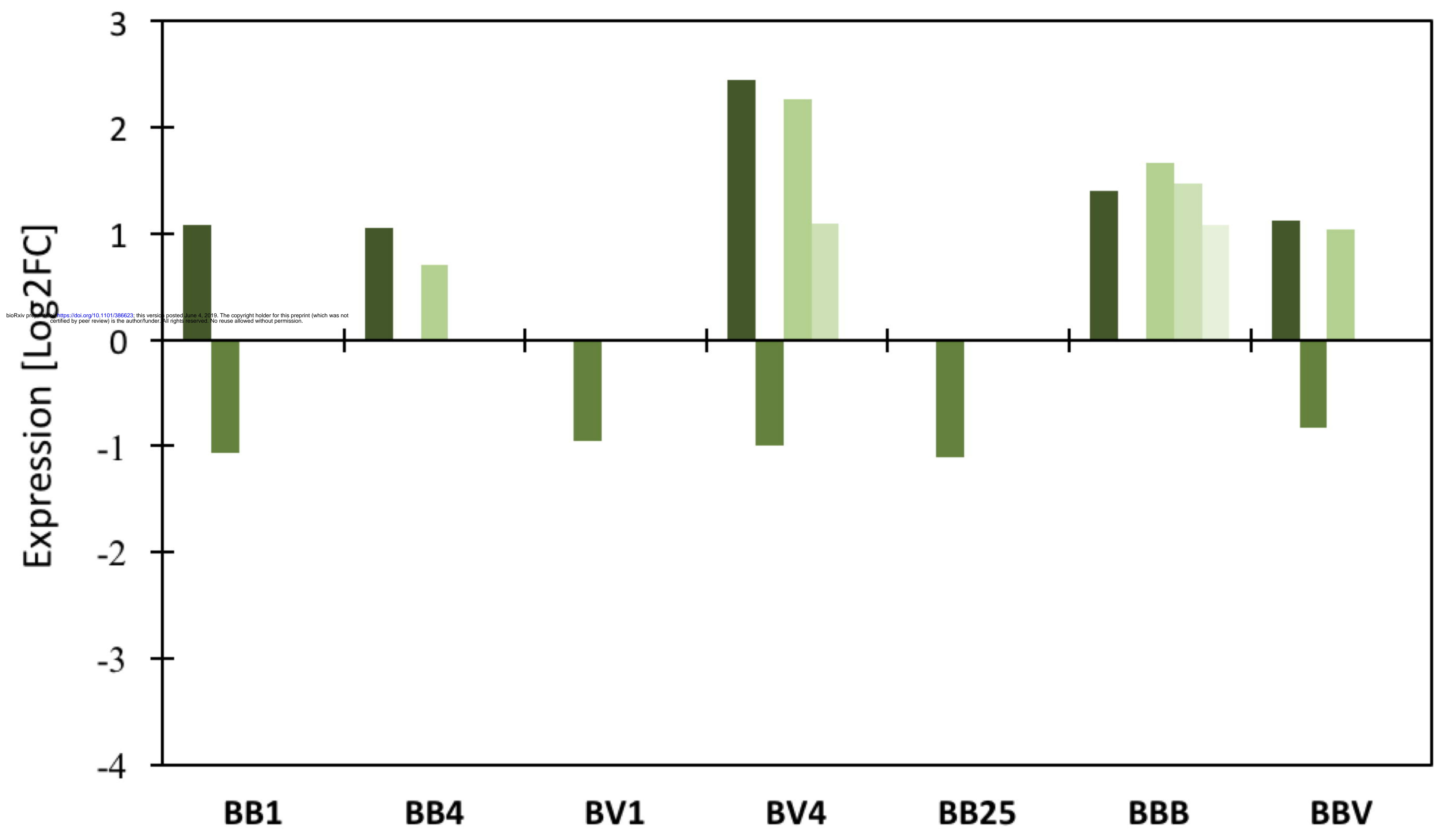

- Biomphamacin 1

Biomphamacin 3

Biomphamacin 4 Biomphamacin 5 Biomphamacin 6 